\title{
Impact of Vanadium(IV)-Oxy Acetylacetonate and Vanadium(III) Acetylacetonatel on the DPPC Liposome Membranes: EPR Studies
}

\author{
D. MAN*, I. MróWKa, A. Wójcik And B. Pytel \\ Institute of Physics, Opole University, Oleska 48, 45-052 Opole, Poland
}

\begin{abstract}
The effect of vanadium (IV)-oxy acetylacetonate (V4) and vanadium(III) acetylacetonate (V3) on the liposome membranes formed of synthetic lecithin (DPPC) was presented in this paper. Liposomes were formed during the sonication of DPPC lecithin in an aqueous medium. The concentration of the vanadium compounds changed in the range of $0 \%$ to $2.4 \%$ in molar ratio to the lecithin. The EPR technique made use of three spin probes penetrating the different areas of the membrane (as follows: TEMPO, 16-DOXYL stearic acid methyl ester, stearic acid 5DOXYL methyl ester). TEMPO probe penetrates the interphase water-lipid (partition parameter $F$ ), the probe 16-DOXYL locates in the middle of the lipid bilayer (rotational correlation time $\tau$ ), the probe 5-DOXYL gives a picture of membrane fluidity (the order parameter) just below the polar head groups. The results of our research showed the following conclusions. The change of membrane fluidity, as a function of admixture concentration, was dependent on the type of additives. TEMPO probe recorded an increase in liquidity interphase water-lipid for both admixtures: V3 and V4. 16-DOXYL probe indicated that the admixture V3 increases the fluidity in the center of the lipid bilayer. Admixture V4 significantly less impacted on the change of the membrane middle. The 5 -DOXYL probe did not influence on the membrane surface portion, there were not observed significant changes under the impact of admixtures. V3 showed stronger impact on membrane fluidity.
\end{abstract}

DOI: $10.12693 /$ APhysPolA.132.52

PACS/topics: 87.16.Dg, 87.64.kh, 87.80.Lg

\section{Introduction}

Vanadium is an element classified as a heavy metal, used in metallurgical industry for producing steel and other alloys. It is also one of the components of organisms and takes part in biochemical processes essential for their activity. Many years of research of vanadium and its impact on biochemical properties, demonstrates us its importance to human life and health [1, 2]. Vanadium was found in all animal cells but its specific biochemical function has not been definitively identified. Scientific research (and different experiments) can suggest that it is one of the essential micronutrients of organisms. For example, the vanadium presence is very important for normal transformation of lipids, phospholipids, and cholesterol [3]. The vanadium affects the shape of red blood cells, moreover, it can stimulate the output of glucose and synthesize glycogen in the liver. Its deficiency can reduce animal growth and the number of red blood cells. It can also decrease calcification of teeth and bones and sometimes can cause abnormal growth of blood lipid level [3]. Vanadium can exist in different oxidation level, ranging from -1 to +5 , but it is most commonly found in the +4 and +5 oxidation level [4,5]. In our experiments liposomes were made from synthetic lecithin DPPC (Fig. 1), containing admixtures of vanadium complexes V4 and V3. V4 - vanadyl

*corresponding author; e-mail: dariusz.man@uni.opole.pl acetylacetonate is the chemical compound of the formula $\mathrm{C}_{10} \mathrm{H}_{14} \mathrm{O}_{5} \mathrm{~V}$. This blue-green complexes consist of the vanadyl group, $\mathrm{VO}^{2+}$, and two acetylacetonate anions, acac $^{-}$. The chemical formula of V3 - vanadium(III) tris (acetylacetonate) is $\mathrm{C}_{15} \mathrm{H}_{21} \mathrm{O}_{6} \mathrm{~V}$. It is a dark yellow to brown powder consists of vanadium group, $\mathrm{VO}^{3+}$, associated with three anions of acetylacetonate. A lot of papers shows that liposomes are a proper model of biological membranes $[6,7]$. Liposomes are susceptible to the biologically active substances $[8,9]$. The best method of the membrane fluidity study is the EPR technique [1012 , because, it permits to investigate the membrane fluidity in transverse section $[13,14]$.

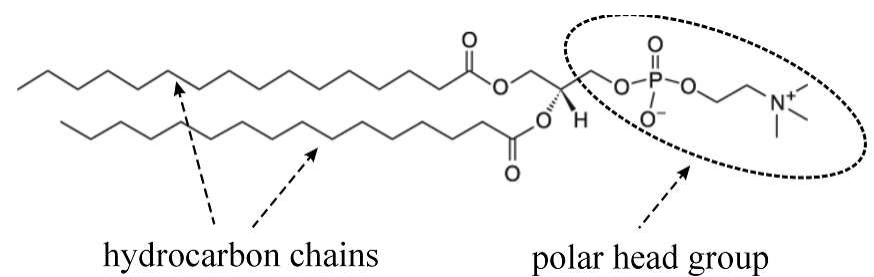

Fig. 1. Schematic structure of lipid molecule dipalmitoylphosphatidylcholine (DPPC) used in the experiment.

\section{Materials and methods}

\subsection{Chemicals}

Pure grade chemicals: vanadium(IV)-oxy acetylacetonate $\mathrm{OV}\left(\mathrm{C}_{5} \mathrm{H}_{7} \mathrm{O}_{2}\right)_{2}$ and vanadium(III) acetylacetonate 
$\mathrm{V}\left(\mathrm{C}_{5} \mathrm{H}_{7} \mathrm{O}_{2}\right)_{3}$ are products by Sigma-Aldrich. Each forms of vanadium were used as admixtures to the liposomes. $20 \%$ of methanol in distilled water was used to prepare the admixtures solution.

\subsection{Liposomes preparation and EPR experiment}

DPPC liposomes were obtained by sonication method in distilled water with the use of an ultrasonic disintegrator TECHPAN UD-20. To avoid overheating each $2 \mathrm{ml}$ sample was sonicated in nine separate cycles: $20 \mathrm{~s}$ of sonication followed by $30 \mathrm{~s}$ of cooling. Total sonication time for a single $2 \mathrm{ml}$ sample was $3 \mathrm{~min}$. The concentration of lecithin in the sample was $80 \mu \mathrm{M}$. For the aqueous dispersion of liposomes, a suitable spin probe was introduced, at a concentration of 1000 ppm in relation to the lecithin. In these researches the EPR spectrometer MX-201R (TU Wrocław, Poland) was used, under the following operating conditions: modulation amplitude $0.016 \mathrm{mT}$, microwave power $60 \mathrm{~mW}$, scanning range $5 \mathrm{mT}$, sweep speed $128 \mathrm{~s}$. EPR spin probe method was used to determine dynamic properties of the liposome membranes. Spin probes were selected depending on its location in different regions of the cross-section of the membrane. Following probes were used in experiment: 2,2,6,6-tetramethylpiperidine1-oxyl (TEMPO), molecular formula $\mathrm{C}_{9} \mathrm{H}_{18} \mathrm{NO}$, 2-ethyl-2-(15-methoxy-15-oxopentadecyl)-4,4-dimethyl3-oxazolidinyloxy (16-DOXYL-stearic acid methyl ester), molecular formula $\mathrm{C}_{23} \mathrm{H}_{44} \mathrm{NO}_{4}$, 2-(4-methoxy4-oxobutyl)-4,4-dimethyl-2-tridecyl-3-oxazolidinyloxy (5-DOXYL-stearic acid methyl ester) (Sigma-Aldrich). TEMPO probe dissolves both in hydrophobic regions of the membrane and aquatic environment (Fig. 2), 5 -DOXYL probe is located under the polar heads of lecithin in the surface region of the membrane (Fig. 2) and 16-DOXYL is placed deep in the hydrophobic area of the membrane (Fig. 2) [13, 15-17]. The partition coefficient of the TEMPO probe between the hydrophobic and hydrophilic phases can be measured by the ratio of signal intensities. In this study the partition coefficient $F$ was measured based on the EPR spectra of TEMPO probe (Fig. 3a). The measure of parameter $F$ is the ratio of the amplitude $P$ of the high-field line in the EPR spectrum of the probe dissolved in water solution to the amplitude of the low-field line in the lipid environment $H$. The value of parameter $F$ is connected, among others, with fluidity of the surface layer of membrane [16].

Based on the spectrum obtained with the 16-DOXYL probe, the spectroscopic parameter $\tau$, rotation correlation time, was determined (Fig. $3 \mathrm{~b}$ ). The value of this parameter also depends on the degree of fluidity of the environment and the higher it is, the stiffer (the more ordered) is the environment in which the probe is immersed [16]. In isotropic environment parameter $\tau$ is a rotational correlation time of the probe. Based on the spectrum obtained using the 5-DOXYL probe, the spectroscopic parameter $T_{I I}$ was determined (Fig. 3c). In-

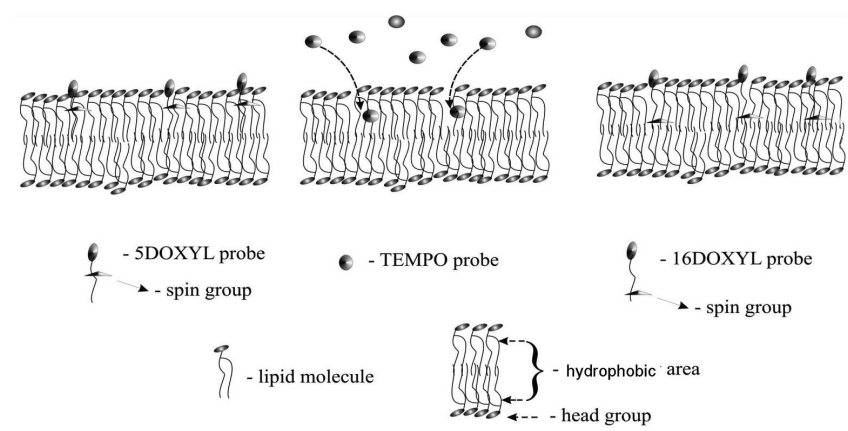

Fig. 2. Schematic structure of liposomes bilayer and location of spin probes used in the experiment.

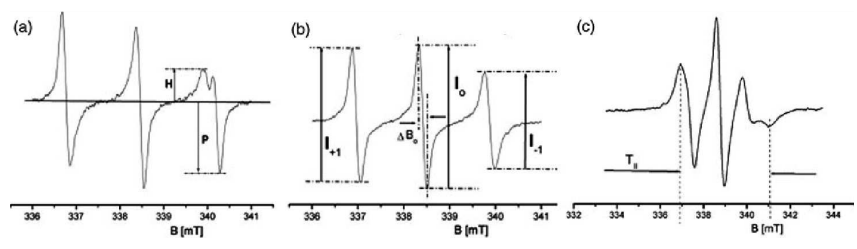

Fig. 3. EPR spectra of spin probes and their spectroscopic parameters: (a) TEMPO, $F=H /(P+H)$; (b) 16-DOXYL-stearic acid methyl ester, $\tau=5.95 \times$ $10^{-10} \Delta B_{0}\left(\sqrt{I_{0} / I_{+1}}+\sqrt{I_{0} / I_{-1}}-1\right) \mathrm{s}$; (c) 5-DOXYLstearic acid methyl ester. Probes were dissolved in an aqueous dispersion of DPPC liposomes.

crease of $T_{I I}$ value means the increase of the order coefficient in the surface region of the membrane [17]. The aqueous solution of liposomes with the spin probe inside was doped with one of the two vanadium compounds V3 and $\mathrm{V} 4$, their concentration were in the range of $0 \%$ to $2.4 \%$ in a molar ratio to the lecithin. Admixture concentrations increased at every step by $0.2 \%$.

The samples were gradually concentrated and vigorously shaken for $120 \mathrm{~s}$. After $120 \mathrm{~s}$ period of stabilization the sample was placed into EPR spectrometer resonant chamber. Sweep time of single sample was $128 \mathrm{~s}$. In order to reduce the uncertainty of measurement, each measurement was repeated three times, and the results were averaged. The relative measurement errors were as follows: $2 \%$ for the $F$ and $T_{I I}$ parameter and $3 \%$ for the $\tau$ parameter.

\section{Results and discussion}

Changing of the fluidity of the liposomes membrane under the influence of complexes of vanadium V3 and $\mathrm{V} 4$ admixtures, the spectroscopic parameters $\left(F, \tau, T_{I I}\right)$ for spin probes (TEMPO, 16-DOXYL, 5-DOXYL) were determined. The results were shown in the graphs, which were presenting the changes in spectral parameters as a function of the concentration of admixtures: $F=f(c)$, $\tau=f(c)$ and $T_{I I}=f(c)$. Figure 4 shows changes the values of the spectroscopic parameter $F$ of probe TEMPO in relation to concentration of admixtures V3 and V4, which were introduced into the aqueous dispersion of liposomes. With the increase of the admixture 
concentration, the value of parameter $F$ increases. This can mean the increase of liquidity of the interface lipidwater. Above $0.8 \%$ concentration of the admixtures in the complexes V3 and V4 there can be seen stabilization of the parameter $F$. It confirmed a maximum of studied complexes solubility in the membranes. V3 admixtures showed higher impact on the liquidity of the interface lipid-water than the V4 ones, which is reflected in the $F$ parameter changes (Fig. 4). Above $1.2 \%$ of the admixture concentration, the spin probe TEMPO was strongly deactivated by the tested complexes (Fig. 5), which prevented continuing the measurements for higher concentrations.

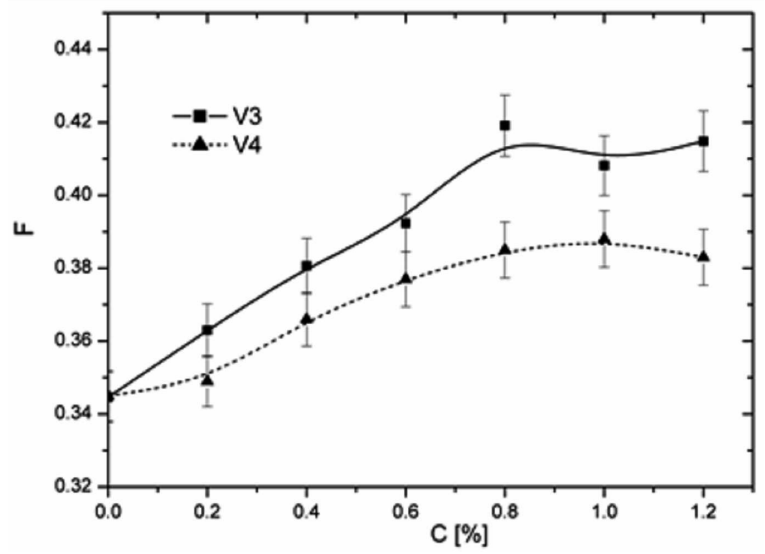

Fig. 4. Effect of the dopant concentration (with respect to the lecithin) on the behavior of the spin probe within the DPPC liposome membrane, based on the measured EPR spectra changes in the partition parameter $(F)$ of the TEMPO probe.

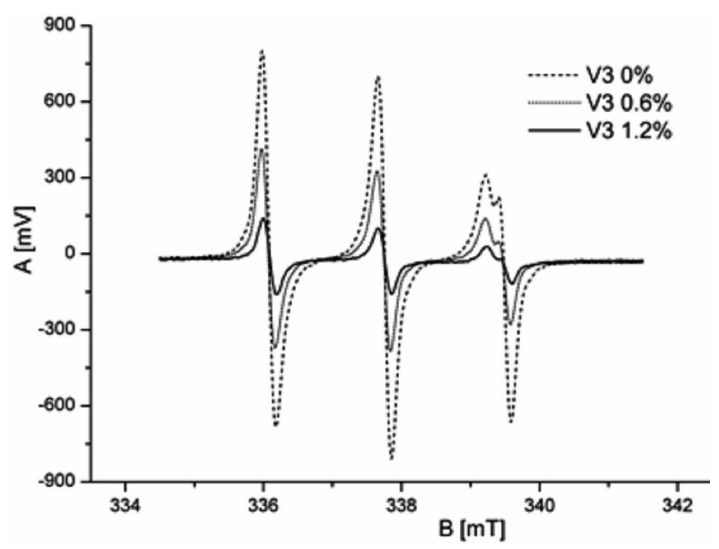

Fig. 5. Shape changes of spectroscopic lines recorder for TEMPO probe placed in the interphase lipid--water part of the DPPC liposome membranes doped by $0.6 \%$ and $1.2 \%$ complex V3.

Figure 6 shows changes values of the spectroscopic parameter $T_{I I}$ of the probe 5 -DOXYL, in relation to concentration of admixtures $\mathrm{V} 3$ and $\mathrm{V} 4$, which were introduced into the aqueous dispersion of liposomes. The data in the chart show that only for admixture V3 we can observe

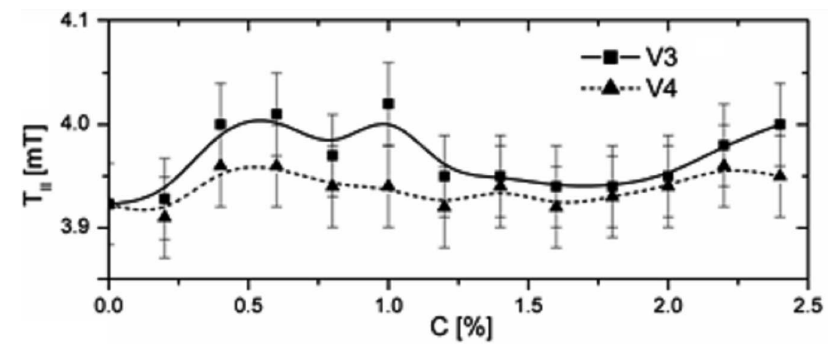

Fig. 6. Effect of the dopant concentration (with respect to the lecithin) on the behavior of the spin probe within the DPPC liposome membrane, based on the measured EPR spectra changes in the parameter $\left(T_{I I}\right)$ of the 5-DOXYL probe.

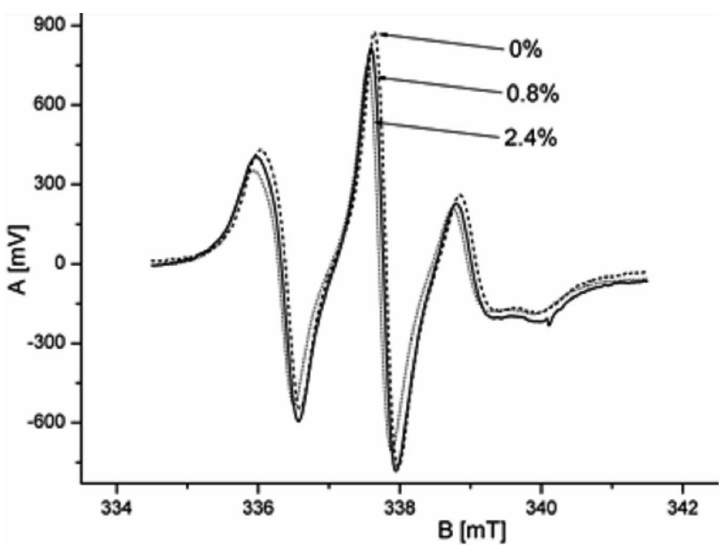

Fig. 7. Changes of spectroscopic lines recorded for 5DOXYL spin probe placed in the under polar head group part of the DPPC liposome membranes doped by $0.8 \%$ and $2.4 \%$ complex V3.

the increase of the parameter $T_{I I}$, with increase of the concentration. The changes reach maximum value in the range of concentration between $0.2 \%$ to $1 \%$. Admixture V4 did not modify the values of parameter $T_{I I}$, which indicated its minimal impact on the liquidity of the surface layer of the membrane.

Figure 7 shows shape changes of spectroscopic lines recorded for 5-DOXYL spin probe placed in the surface

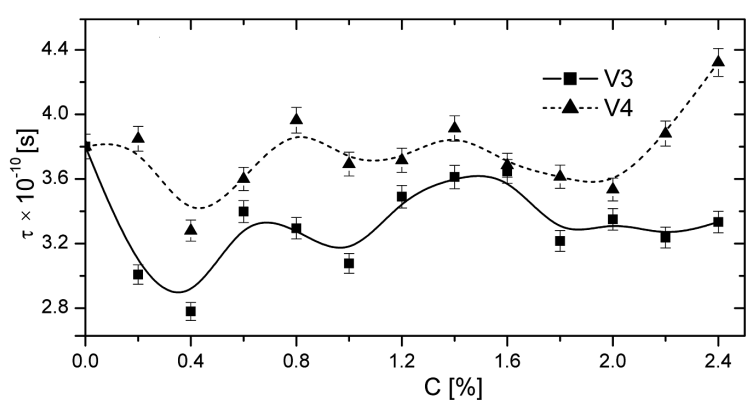

Fig. 8. Effect of the dopant concentration (with respect to the lecithin) on the behavior of the spin probe within the DPPC liposome membrane, based on the measured EPR spectra changes in the parameter $(\tau)$ of the 16-DOXYL probe. 


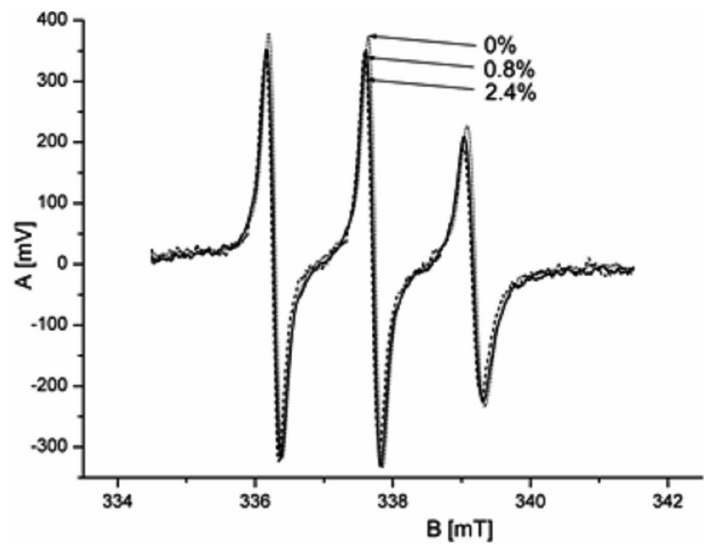

Fig. 9. Shape changes of spectroscopic lines recorded for 16-DOXYL spin probe placed in the central part of the DPPC liposome membranes doped by $0.8 \%$ and $2.4 \%$ complex V3.

region of the membrane, doped by $0.8 \%$ and $2.4 \%$ complex V3.

Figure 8 showed the changes of the values of spectroscopic parameter of probe 16-DOXYL in admixtures V3 and V4 concentration introduced to the aqueous dispersion of liposomes. The data (presented in the graph) confirmed that the concentration range between $0 \%$ to $0.4 \%$ consequently gives strong decrease of the value of the parameter $\tau$. Above $0.6 \%$ concentration of admixture the parameter value $\tau$ begins to oscillate: for $\mathrm{V} 4$ around the initial value $\left(\tau_{0}\right)$, for $\mathrm{V} 3$ around the value is slightly lower than $\tau_{0}$. Above $1.8 \% \mathrm{~V} 3$ admixtures concentration the parameter $\tau$ stabilized around $\tau=3.3$, whereas in case of admixtures V4 the strong increase of the value of the parameter $\tau$ was observed. The impact such as of the probe in the middle of the lipid bilayer confirmed the membranes liquefaction by admixtures V3, and reducing membranes liquidity by admixtures V4 in concentration above $1.8 \%$.

Figure 9 shows shape changes of spectroscopic lines recorded for 16-DOXYL spin probe placed in the central part of the DPPC liposome membranes doped by $0.8 \%$ and $2.4 \%$ complex V3.

\section{Conclusion}

The tested vanadium compounds have been changing the membrane fluidity of liposomes in a similar way. V3 complex in all studies showed higher activity than V4 complex in action on the membrane of liposomes. It can be associated with a better dissolution of V3 compounds in a hydrophilic condition. Its biggest impact was observed on the interface water-lipid which showed changes in the value of the parameter $F$ for the probe TEMPO (Fig. 4). The increase of the fluidity in this part of membranes under the impact of both V3 and V4 was observed. The membrane part below the polar head groups (hydrophobic part) was the less sensitive to admixtures $\mathrm{V} 3$ and V4. The minimal impact on the parameter $T_{I I}$ change was observed (Fig. 6). Under the influence V3 compounds fluidity of the middle part of the lipid membrane has increased. However, for V4 compounds fluidity has decreased above $2 \%$ concentration of compounds V4 (Fig. 8). This behavior of V4 compound (in higher concentration) was connected with a better solubility in a hydrophobic medium compared with V3 compound. Probably the V4 concentrations above $2 \%$, attracted more intensive on the hydrocarbon chains of phospholipids - the part of molecules and inhibited their vibration, whereas V3 compound inside a strong hydrophobic medium dissolves very poorly, which prevents its stronger impact on the fluidity of the bilayer.

\section{References}

[1] D.G. Barceloux, D. Barceloux, J. Toxicol. Clin. Toxicol. 37, 265 (1999).

[2] D. Sanna, V.L. Pecoraro, G. Micera, E. Garribba, J. Biol. Inorg. Chem. 17, 773 (2012).

[3] B.F. Harland, B.A. Harden-Williams, 0.1016/00028223(94)92371-XJ. Am. Diet. Assoc. 94, 891 (1994).

[4] P. Galloni, V. Conte, B. Floris, Coordin. Chem. Rev. 301-302, 240 (2015)

[5] Y. Liu, Q. Zhou, J. Xu, Y. Xue, X. Liu, J. Wang, C. Xue, Environ. Geochem. Health 38, 111 (2016).

[6] C. Matos, C. Moutinho, P. Lobăo, J. Membrane Biol. 245, 69 (2012).

[7] G. Sessa, G. Weissmann, J. Lipid Res. 9, 310 (1968).

[8] E. Boniewska-Bernacka, D. Man, R. Słota, M.A. Broda, J. Biochem. Mol. Toxic. 25, 231 (2011).

[9] S. Mitrus, D. Man, J. Biochem. Mol. Toxic. 26, 162 (2012).

[10] D. Man, J. Lipos. Res. 18, 225 (2008)

[11] D. Man, R. Słota, M.A. Broda, G. Mele, J. Li, J. Biol. Inorg. Chem. 16, 173 (2011).

[12] R. Wałęsa, D. Man, G. Engel, D. Siodłak, T. Kupka, T. Ptak, M.A. Broda, Chem. Biodivers. 12, 1007 (2015).

[13] D. Man, R. Olchawa, J. Lipos. Res. 23, 327 (2013).

[14] R. Olchawa, D. Man, B. Pytel, Nukleonika 60, 395 (2015).

[15] E.J. Shimshick, H.M. McConnell, Biochemistry 12 2351 (1973).

[16] M.A. Hemminga, Chem. Phys. Lipids 32, 323 (1983).

[17] S. Schreier, C.F. Polnaszek, I.C.P. Smith, Biochim. Biophys. Acta Rev. Biomembranes $\mathbf{5 1 5}$ 375 (1978). 